\title{
Dexmedetomidine protects aged rats from postoperative cognitive dysfunction by alleviating hippocampal inflammation
}

\author{
NIANPING CHEN $^{1 *}$, XIUFANG CHEN $^{2 *}$, JIANPING XIE $^{2}$, CHENGLONG WU $^{2}$ and JIANG QIAN ${ }^{3}$ \\ ${ }^{1}$ Department of Anesthesiology, Affiliated Hospital of Shaoxing University (Shaoxing Municipal Hospital); \\ ${ }^{2}$ Department of Neurology, People's Hospital of Shaoxing, Shaoxing, Zhejiang 312000; \\ ${ }^{3}$ Department of Anesthesiology, Zhejiang Hospital, Hangzhou, Zhejiang 310013, P.R. China
}

Received November 3, 2018; Accepted May 3, 2019

DOI: $10.3892 / \mathrm{mmr} .2019 .10438$

\begin{abstract}
The present study investigated the effect of dexmedetomidine on hippocampal inflammation and cognitive function in rats with postoperative cognitive dysfunction (POCD). A total of 80 healthy male Sprague Dawley rats were used, 72 of which developed POCD. The rats were randomly divided into four groups: The control, model, low-dose and high-dose dexmedetomidine anesthesia groups. A POCD model was established and dexmedetomidine was administered. Cognitive function tests were performed and expression levels of interleukin $1 \beta$ (IL-1 $\beta$ ), tumor necrosis factor $\alpha(\mathrm{TNF}-\alpha)$ and NF- $\alpha \mathrm{B}$ biomarkers were evaluated on the first, third and seventh day following modeling. The cognitive function of rats was measured using a Y-maze test. The expression levels of IL- $1 \beta$ and TNF- $\alpha$ in the hippocampus were determined by ELISA. The protein expression levels of $\mathrm{NF}-\kappa \mathrm{B}$ p65 in the hippocampus were determined by western blotting. It was revealed that at 1, 3 and 7 days after surgery, there were no alterations in the exercise ability of rats in the different groups, as reflected by the number of rats passing the alternative arms in the Y-maze. On the first and third day after surgery, the cognitive dysfunction reflected by the alteration scores of the low-dose and high-dose dexmedetomidine anesthesia groups were significantly higher than those of the model group, and the increase in the high-dose group was more pronounced. Additionally, on the first day after surgery, the expression levels of IL- $1 \beta$, TNF- $\alpha$ and NF- $\kappa$ B in the hippocampi of rats in the low- and high-dose dexmedetomidine anesthesia groups were significantly lower than those in the model group, and the decrease was more pronounced
\end{abstract}

Correspondence to: Dr Jiang Qian, Department of Anesthesiology, Zhejiang Hospital, 12 Lingyin Road, Hangzhou, Zhejiang 310013, P.R. China

E-mail: qianjiangsx@163.com

*Contributed equally

Key words: dexmedetomidine, postoperative cognitive dysfunction, inflammatory factors, microglia in the high-dose group. At 7 days after surgery, the differences in expression levels of IL- $1 \beta$, TNF- $\alpha$ and NF- $\kappa$ B in the hippocampus among groups were not identified to be statistically significantly different. Taken together, the results of the present study indicated that dexmedetomidine may inhibit hippocampal inflammation induced by surgical trauma, and that dexmedetomidine may effectively improve postoperative cognitive function in rats.

\section{Introduction}

The occurrence of postoperative cognitive dysfunction (POCD) is closely associated with the inflammatory response of the central nervous system (CNS) (1). POCD is characterized by progressive deterioration of cognitive function and reduced self-care ability. Permanent cognitive disabilities can develop into neurodegenerative diseases similar to Alzheimer's disease and dementia, and may lead to the loss of independent living abilities (2). Trauma caused by surgery is regarded as a major cause of POCD in the senior population (3), but its neurobiological basis is largely unknown. A previous study demonstrated that the learning ability of mice is reduced, and interleukin $1 \beta$ (IL-1 $\beta$ ) expression in the hippocampus is significantly increased following lipopolysaccharide (LPS) injection into the peripheral blood of mice (4). Cao et al (5) and Wuri et al (6) separately performed partial hepatectomies on rats and mice; they found decreased learning and memory ability, and increased inflammatory factors in the hippocampus in the postoperative animals $(5,6)$.

Dexmedetomidine is a commonly used $\alpha 2$ receptor agonist with sedative and analgesic effects. Its $\alpha 2: \alpha 1$ ratio is 1,600:1, and its affinity is eight times greater than that of clonidine (7). It produces sedative, hypnotic and anxiolytic effects by acting on $\alpha 2$ receptors in the locus coeruleus of the brain stem (8). A previous study revealed that dexmedetomidine exhibits anti-inflammatory effects at multiple sites (9), including in lung, kidney and rat sepsis models. It exhibits neuroprotective effects in animal models, including ischemic brain injury and spinal cord injury models (10). Additionally, a previous study demonstrated that dexmedetomidine may inhibit inflammation caused by LPS-induced microglial activation (11). A previous study indicated that postoperative cognitive impairment is associated with inflammation of the CNS (12). The activation 
of microglia and NF- $\mathrm{KB}$ can lead to overexpression of inflammatory factors, including tumor necrosis factor $\alpha(\mathrm{TNF}-\alpha)$ and IL-1 $\beta$, which may cause postoperative cognitive decline (13). $\mathrm{NF}-\mathrm{\kappa B}$ is involved in the regulation of neuroinflammation, such as that associated with cerebral ischemia and hypoxia. It is a key molecule of the inflammatory response, which mediates the expression of inflammatory factors in multiple signaling pathways (14). A previous study demonstrated that activated NF- $\mathrm{KB}$ can induce the expression of inflammatory factors in microglia (15).

In the present study, a model of hepatectomy in rats was selected to simulate cognitive dysfunction following surgery. The effect of dexmedetomidine on the expression levels of TNF- $\alpha$, IL- $1 \beta$ and NF- $\kappa B$ in the hippocampus was examined to explore whether dexmedetomidine may inhibit the inflammatory response in the CNS, and its possible mechanism of action.

\section{Materials and methods}

Ethics approval. The present study was approved by the Institutional Animal Care and Use Committee of Zhejiang Hospital.

Establishment of a rat model of POCD. A partial hepatectomy was used to establish a POCD model in aged rats. A total of 80 male Sprague Dawley rats (age, 18 months; weight, $500-600 \mathrm{~g}$ ) were provided by the Experimental Animal Center of Zhejiang Hospital. The animals were housed at $22 \pm 2^{\circ} \mathrm{C}$, a relative humidity of $45-75 \%$ and with a $12 \mathrm{~h}$ light-dark cycle. Food and water were freely accessible. Preoperative fasting was carried out for $12 \mathrm{~h}$. The rats were administered inhaled isoflurane (1.5-2.0\%) for anesthesia. Tracheal intubation was performed and mechanical ventilation was used in the rats. The rats were anesthetized throughout the operation by exposure to a mixture of air $\left(21 \% \mathrm{O}_{2}\right.$ with $\left.79 \% \mathrm{~N}_{2}\right)$ and $1.5-2.0 \%$ isoflurane. Following disinfection, a $3-\mathrm{cm}$ vertical incision was made at the lower edge of the xiphoid process, and the left hepatic lobe was dissected. The left hepatic lobe was probed and ligated to the root, and subsequently resected. The incision was infiltrated with $0.25 \%$ bupivacaine (Nanjing Chemlin Chemical Industry Co., Ltd.) and sutured with a 3-0 suture. The procedure was performed aseptically and the surgery time was controlled at $\sim 30 \mathrm{~min}$. After the rats returned to consciousness, they were returned to the animal room.

Animal grouping. The upper limit of the $95 \%$ CI of the mean training duration of rats in the Y-maze test was used to decide whether the rats that had undergone surgery had cognitive dysfunction, according to a previously described method (16). Among the 80 rats that received hepatectomy, 72 rats developed POCD. These 72 rats were randomly divided into four groups: i) Blank control group; ii) low-dose anesthesia group; iii) high-dose anesthesia group; and iv) surgery model group. The blank control group was not treated. In a transparent anesthetic chamber, rats were exposed for $4 \mathrm{~h}$ to $1.5 \%$ isoflurane (Baxter) in $21 / \mathrm{min}$ of $100 \%$ oxygen (Renyuan Chem) as the carrying gas, or to vehicle ( $2 \mathrm{l} / \mathrm{min}$ of $100 \%$ oxygen), $24 \mathrm{~h}$ after hepatectomy. At the outlet of the chamber, the gas composition (concentrations of isoflurane, oxygen and $\mathrm{CO}_{2}$ ) within the chamber was continuously analyzed by a gas monitor (Datex-Ohmeda; GE Healthcare). Following anesthesia, the rats received $100 \%$ oxygen until they recovered to consciousness. The heart rate and oxygen saturation of the rats were monitored to maintain normal heart rate and $>90 \%$ oxygen saturation during surgery. Dexmedetomidine (10 and $30 \mu \mathrm{g} / \mathrm{kg}$; Nanjing Chemlin Chemical Industry Co., Ltd.) was intraperitoneally injected $1 \mathrm{~h}$ after surgery for the low- and high-dose anesthesia groups, respectively. The dose selection was based on previous studies $(17,18)$. All rats received an intramuscular injection of penicillin (200,000 units) daily for 3 days after surgery to prevent infection. A total of 6 rats were taken from each group on the 1st, 3rd and 7th day after treatment to perform a Y-maze test. Following anesthesia with $2 \%$ pentobarbital sodium $(50 \mathrm{mg} / \mathrm{kg}$; intraperitoneal), the hippocampus was quickly dissected and separately immersed into liquid nitrogen and maintained at $-80^{\circ} \mathrm{C}$ prior to analysis. Peripheral blood was collected and placed into an anticoagulant tube. Following the intervention, the rats were sacrificed by cervical dislocation. The supernatant of the solution in the anticoagulant tube was removed by centrifugation $(8,000 \mathrm{xg}$ for $20 \mathrm{~min}$ at $4^{\circ} \mathrm{C}$ ). The extracted hippocampal tissues and supernatants were stored in a refrigerator at $-80^{\circ} \mathrm{C}$ for subsequent use.

Behavioral testing. A behavior test (Y-maze) was generally designed to confirm whether POCD developed in rats suffering from surgical trauma. A total of 6 rats were taken from each group at 1,3 and 7 days after treatment, and a Y-maze test was performed between 9:00 and 12:00 a.m. The Y-maze test was performed using a previously described method with minor modification (19). The Y-maze was divided into three arms, labeled as I, II and III, and the angle between the three arms was $120^{\circ}$. The size of each arm was $40 \times 30 \times 15 \mathrm{~cm}$, and the length of the equilateral triangle encircled by the three arms was $15 \mathrm{~cm}$. The light source and camera were set $2 \mathrm{~m}$ above the center of the Y maze. A cloth was installed on the outside of the maze to prevent external light interference. The three arms of the Y maze were randomly set as start arm, novel arm and the other arm. Prior to testing, the novel arm was blocked with a septum, and the rat was placed in the start arm and allowed to move freely in the start arm and the other arm. The rat was removed after $10 \mathrm{~min}$ of adaptation. After $1 \mathrm{~h}$, the test was started: The novel arm was opened, and the rat was placed in the start arm and allowed to freely move among the three arms for $5 \mathrm{~min}$. The shuttle path was recorded. At the bottom of the labyrinth, wood chips were placed on the bottom of the maze. Following each training, the wood chips in the three arms were mixed and re-paved. After each test, the labyrinth was cleaned to remove odors and residues to prevent any influence on the test results. In the experiment, different rats were randomly arranged with different start, novel and other arms. During the training and testing of the same rat, the settings were not changed. The entire process of the test was observed and recorded by the camera directly above the maze. The motion path of test was recorded, and the total entries and the spontaneous alternation score were calculated. The calculation method was as follows: Assume that the novel arm is $\mathrm{A}$, the start arm is B, and the other arm is C. For example, the motion path during the test of the rat is BACABCBAB. During 
the test, the rat passes through 9 arms, thus the number of shuttles is 9. Continuous $\mathrm{ABC}, \mathrm{BCA}$ and $\mathrm{CAB}$ were recorded as 1 point at a time. In this example, there were $\mathrm{BAC}, \mathrm{CAB}$, $\mathrm{ABC}$ and $\mathrm{CBA}$; the alternation score was 4 , and the alternation ratio formula was: The spontaneous alternation score $(\%)=[($ number of alternation $) /($ total arm entries -2$)] \times 100 \%$. In this example, the alternation scoring rate $=4 / 7 \times 100 \%=57.14 \%$. The total number of passing arms in the Y-maze is considered as an index reflecting the exercise ability of the rats.

ELISA for detection of $I L-1 \beta$ and $T N F-\alpha$. An ELISA was used to detect the expression levels of IL-1 $\beta$ and TNF- $\alpha$ in hippocampal tissues. Frozen hippocampus samples from four POCD rats from each group were mixed with an appropriate amount of physiological saline. They were fully homogenized and centrifuged at a speed of $8,000 \mathrm{x}$ for $10 \mathrm{~min}$ at $4^{\circ} \mathrm{C}$. The supernatant was collected and tested using a rat IL-1 $\beta$ ELISA kit (cat. no. ab100768; Abcam) or a rat TNF- $\alpha$ ELISA kit (cat. no. ab100785; Abcam), according to the manufacturer's protocol.

Determining $N F-\kappa B$ p65 expression in the hippocampus by western blotting. Protein extraction was performed using RIPA buffer and a protease inhibitor cocktail [1.4\% SDS, $2 \%$ dithiothreitol, $120 \mathrm{mM}$ Tris- $\mathrm{Cl}$ (pH 6.8), $7 \mathrm{M}$ urea, $2 \mathrm{M}$ sulfourea, $20 \mathrm{mM}$ DTT, $3.20 \mathrm{mM}$ Tris (pH 7.5), $1.5 \mathrm{mM} \mathrm{NaCl}$, 1 mM EDTA, 1 mM EGTA, $1 \%$ Triton X-100, 2.5 mM sodium pyrophosphate, $1 \mathrm{mM} \beta$-glycerophosphate]. A bicinchoninic acid assay kit (Sigma-Aldrich; Merck KGaA) was used to determine protein concentration in samples from four POCD rats. After sample buffer was added, the samples were boiled at $95^{\circ} \mathrm{C}$ for $10 \mathrm{~min}$. Then, the proteins $(30 \mu \mathrm{g} / \mathrm{sample} / \mathrm{well})$ were separated using $10 \%$ polyacrylamide gel electrophoresis. After electrophoresis, proteins were transferred onto PVDF membranes at $100 \mathrm{~V}$ transfer-molded voltage for 45-70 min. Subsequently, samples were incubated at room temperature for $1 \mathrm{~h}$ with 5\% BSA (Shanghai Rebiosci Biotechnology Co., Ltd.), and then incubated with rabbit anti-NF- $\mathrm{B} / \mathrm{p} 65$ (1:1,000; cat. no. ab16502; Abcam) and mouse anti- $\beta$-actin primary antibodies (1:1,000; cat. no. ab8226; Abcam), at $4^{\circ} \mathrm{C}$ overnight. Following that, samples were washed with TBS with Tween-20 three times (5 min each). A horseradish peroxidase secondary antibody (1:3,000; cat. no. ab6721; Abcam) was added for incubation at room temperature for $1 \mathrm{~h}$. Subsequently, membranes were washed three times (5 min/time). Development was performed using SuperSignal West Femto chemiluminescence reagent (Thermo Fisher Scientific, Inc). $\beta$-actin was used as an internal reference. Bands were visualized with a Bio-Rad Gel Doc EZ imager (Bio-Rad Laboratories, Inc.). ImageJ software (version 1.8.0_112; National Institutes of Health) was used to analyze the intensity of the target bands.

Immunofluorescent staining for detection of activation of microglial cells. Ionized calcium-binding adapter molecule 1 (Iba-1) is a biomarker of microglia and macrophages (20). By labeling microglia with Iba-1 antibody, the activated microglia may be quantified. Frozen hippocampal tissues were cut into 2-3 mm slices and loaded into embedding cassettes. The tissues were submerged and fixed in a solution of neutral buffered formalin for at least $48 \mathrm{~h}$ at room temperature. When using zinc fixatives (formalin-zinc sulfate fixative; Beijing Solarbio Science and Technology Co., Ltd.), the same sized tissues (2-3 mm), were fixed in a zinc fixation buffer for $24 \mathrm{~h}$ at room temperature. Tissues were then fixed in paraffin for 10-15 min at room temperature. The paraffin embedded sections of hippocampal tissue of four POCD rats from each group were dewaxed, hydrated and antigen-repaired. To enhance the accessibility of the antibody to antigens, antigen retrieval was performed prior to incubation with the primary antibody. Briefly, slides were placed in citrate buffer in a slide container and the container was placed in a metal container filled with distilled water in a decloaker. The decloaker was heated to a temperature of $122^{\circ} \mathrm{C}$ and was allowed to naturally air cool to $89^{\circ} \mathrm{C}$. The lid was then opened for further cooling. Slides were blocked using 5\% goat serum (Abcam) in PBS for $10 \mathrm{~min}$ at room temperature and then incubated with primary anti-Iba-1 (1:500; cat. no. ab178847; Abcam) at $4^{\circ} \mathrm{C}$ overnight. Following incubation with fluorescent secondary antibody (AlexaFluor488-conjugated anti-rabbit; 1:1,000; cat. no. A27034; Invitrogen; Thermo Fisher Scientific, Inc.) for $2 \mathrm{~h}$ in the dark, the nucleus was stained with DAPI $(1 \mu \mathrm{g} / \mathrm{ml})$ and mounted for $10 \mathrm{~min}$ at room temperature, and observed under a confocal fluorescence microscope (magnification, $\mathrm{x} 400$ ). A total of five independent fields were selected and the activated microglia and total microglia in the visual field were counted: Microglial activation rate $=$ activated microglia number/total microglia number $\times 100 \%$. The microglial activation rates from five fields were averaged as the microglial activation rate of the specimen.

Statistical analysis. All data were analyzed by GraphPad Prism version 6 statistical software (GraphPad Software, Inc.). Each experiment was repeated three times. Measurement data are presented as the mean \pm SD. One-way ANOVA followed by a Least Significant Difference post hoc test was applied for comparisons among multiple groups. $\mathrm{P}<0.05$ was considered to indicate a statistically significant difference.

\section{Results}

Alterations in the cognitive function of rats in each group. The total number of arms a rat passes in the Y-maze test is considered to be an index reflecting the exercise ability of the rats (17). The number of rats passing alternative arms at each time point is shown in Fig. 1. There was no statistical difference in the number of rats passing alternative arms in each group at 1, 3 or 7 days after surgery. This indicated that neither anesthesia nor partial hepatectomy affected the exercise activity of rats. The alternation scoring rate of each group of rats at 1 , 3 and 7 days is shown in Fig. 2. The alternation score of the model group was significantly lower than that of the control group at 1 and 3 days $(\mathrm{P}<0.05)$. Compared with the model group, the alternation scores of the high-dose and low-dose anesthesia groups were significantly higher at 1 and 3 days $(\mathrm{P}<0.05)$, particularly in the high-dose group. On the seventh day, there was no statistically significant difference identified in the alternation score among the rats in each group ( $\mathrm{P}>0.05)$. These results indicated that anesthesia and surgery did not alter the exercise ability of the rats, but decreased the cognitive function of the rats. 




Figure 1. Number of passes between alternative arms in the Y-maze in each group at different time points following surgery. There was no statistically significant difference observed in the number of rats passing the alternative arms in each group at 1, 3 or 7 days after surgery.

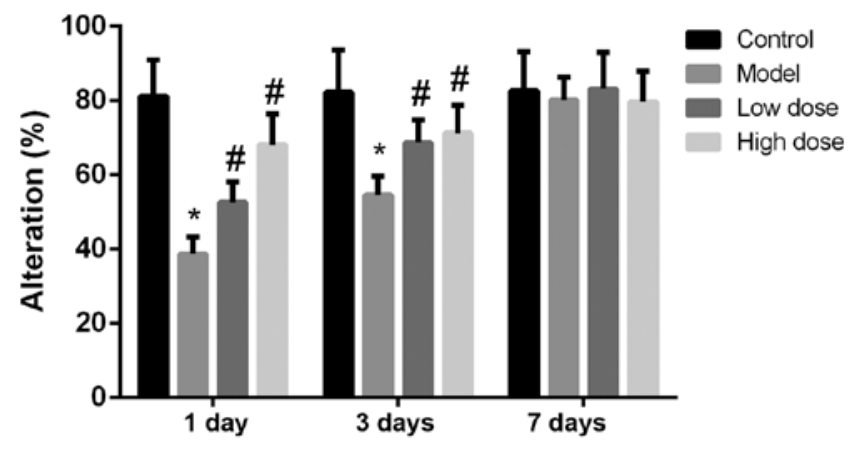

Figure 2. Alteration scores at different time points following surgery. The alteration score of the model group was significantly lower than that of the control group. The alteration scores of the high-dose and low-dose anesthesia groups were significantly higher compared with those of the model group, particularly in the high-dose group. On day 7, there was no statistical difference in alteration score among rats in each group. ${ }^{*} \mathrm{P}<0.05$ vs. control group; ${ }^{\#} \mathrm{P}<0.05$ vs. model group.

Anesthesia and surgery lead to decreased expression levels of $I L-1 \beta$ and $T N F-\alpha$ in the rat hippocampus. The expression levels of IL-1 $\beta$ and TNF- $\alpha$ in the hippocampi of rats from each group were detected by ELISA. As shown in Fig. 3, at 1 and 3 days after surgery, the expression levels of IL-1 $\beta$ and TNF- $\alpha$ in the model group were significantly higher than those in the control group $(\mathrm{P}<0.05)$. The expression levels of IL-1 $\beta$ and TNF- $\alpha$ in the high-dose and low-dose anesthesia groups decreased significantly $(\mathrm{P}<0.05)$, and the drop in the high-dose group was slightly more pronounced. On day 7 after surgery, the expression levels of IL-1 $\beta$ and TNF- $\alpha$ were low in all groups and there were no significant differences identified among the groups $(\mathrm{P}>0.05)$.

Expression levels of $N F-\kappa B$ p65 in rat hippocampus tissues at each time point for each group. The expression levels of NF- $\mathrm{B}$ p65 in rat hippocampus tissues were detected by western blotting (Fig. 4). NF- $\kappa \mathrm{B}$ p65 expression in the model group was significantly increased compared with the control group at 1 and 3 days after surgery $(\mathrm{P}<0.05)$. The expression levels of NF- $\kappa$ B p65 in the high-dose and low-dose groups were significantly decreased compared with the model group $(\mathrm{P}<0.05)$, and the decrease in the high-dose group was more obvious. At 7 days after surgery, the expression levels of
$\mathrm{NF}-\kappa \mathrm{B}$ p65 relatively low and there was no significant difference identified among the groups $(\mathrm{P}>0.05)$.

Activation of microglial cells in rat brains. By labeling microglia with Iba-1 antibody, activated microglia may be quantified. The Iba-1 antibody immunofluorescence staining results are shown in Fig. 5. At 1 and 3 days after surgery, the microglial cells in the brain tissues of rats in the control group were in an inactive state, the cell bodies were small and irregular protrusions were observed. By contrast, the microglial cells in the brain tissues of rats in the model group were large, the cell bodies were round, and they exhibited an activation state of deformation and phagocytosis. On day 1 , the quantification of immunofluorescence staining revealed that the proportion of activated microglial cells was $79.54 \pm 5.52 \%$ in the model group, which was significantly higher than that of the control group $(\mathrm{P}<0.05)$. The activation of microglia in brain tissue from the high-dose and low-dose anesthesia groups was inhibited, and the activation rates were $21.84 \pm 2.03$ and $39.45 \pm 3.55 \%$, respectively, which were significantly lower than that of the model group $(\mathrm{P}<0.05)$. The activation of microglia in brain tissue was decreased to $55.16 \pm 5.23 \%$ in the model group and to $15.97 \pm 1.26 \%$ (low-dose) and $10.65 \pm 1.12 \%$ (high dose), respectively, at 3 days after surgery. Microglia activation in the low-dose and high-dose groups was significantly lower compared with the model group at 1 and 3 days. At 7 days after surgery, there was no statistically significant difference observed in the activation rates of microglial cells in the brain tissues of each group $(\mathrm{P}>0.05)$. These results indicated that microglia were activated by dexmedetomidine.

\section{Discussion}

Tissue injury caused by surgical trauma leads to the activation of the immune system, and release of inflammatory factors (21). The amount of inflammatory factor release is associated with the degree of surgical trauma (22). To understand whether surgical trauma could affect cognitive function in rats, a partial hepatectomy was performed and a Y-maze test was conducted. The results revealed a non-significant difference in the total entry numbers of rats passing the Y-maze in the control and model groups. However, the alternation score of rats in the model group was significantly lower than that of the control group at 1 and 3 days after surgery, and dexmedetomidine treatment significantly increased this score. At 7 days, the alternation score in rats of all groups returned to the level of the control group. These results indicated that cognitive function was decreased following hepatectomy, and dexmedetomidine could protect postoperative cognitive function in the short term.

Under physiological conditions, the presence of the blood-brain barrier prevents the entry of macromolecule proteins into brain tissue. However, the permeability of the blood-brain barrier increases under certain circumstances, such as in ischemia, trauma, tumors, neurological conditions associated with neuronal hyper-excitability, epileptic seizures and spreading depolarizations. In the case of POCD, a large number of inflammatory factors and immune cells from the peripheral blood can enter into the brain (23). A previous study demonstrated that cutting the glossopharyngeal nerve 

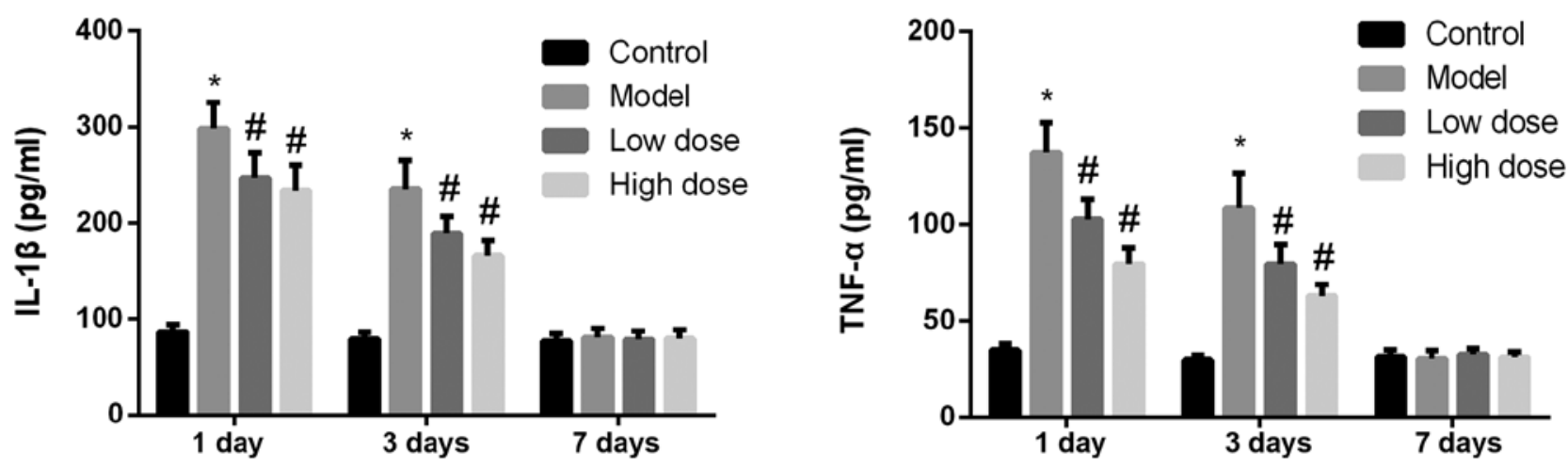

Figure 3. Expression levels of IL-1 $\beta$ and TNF- $\alpha$ in the rat hippocampus following surgery. At 1 and 3 days after surgery, the expression levels of IL-1 $\beta$ and TNF- $\alpha$ in the model group were significantly higher compared with those in the control group. The expression levels of IL-1 $\beta$ and TNF- $\alpha$ in the high-dose and low-dose anesthesia groups were decreased significantly compared with in the model group, and the decrease in the high-dose group was more pronounced. At 7 days after surgery, the expression levels of IL- $1 \beta$ and TNF- $\alpha$ were low in all groups and there was no statistically significant difference identified among the groups. ${ }^{*} \mathrm{P}<0.05$ vs. control group; ${ }^{\#} \mathrm{P}<0.05$ vs. model group. IL-1 $\beta$, interleukin $1 \beta$; TNF- $\alpha$, tumor necrosis factor $\alpha$.
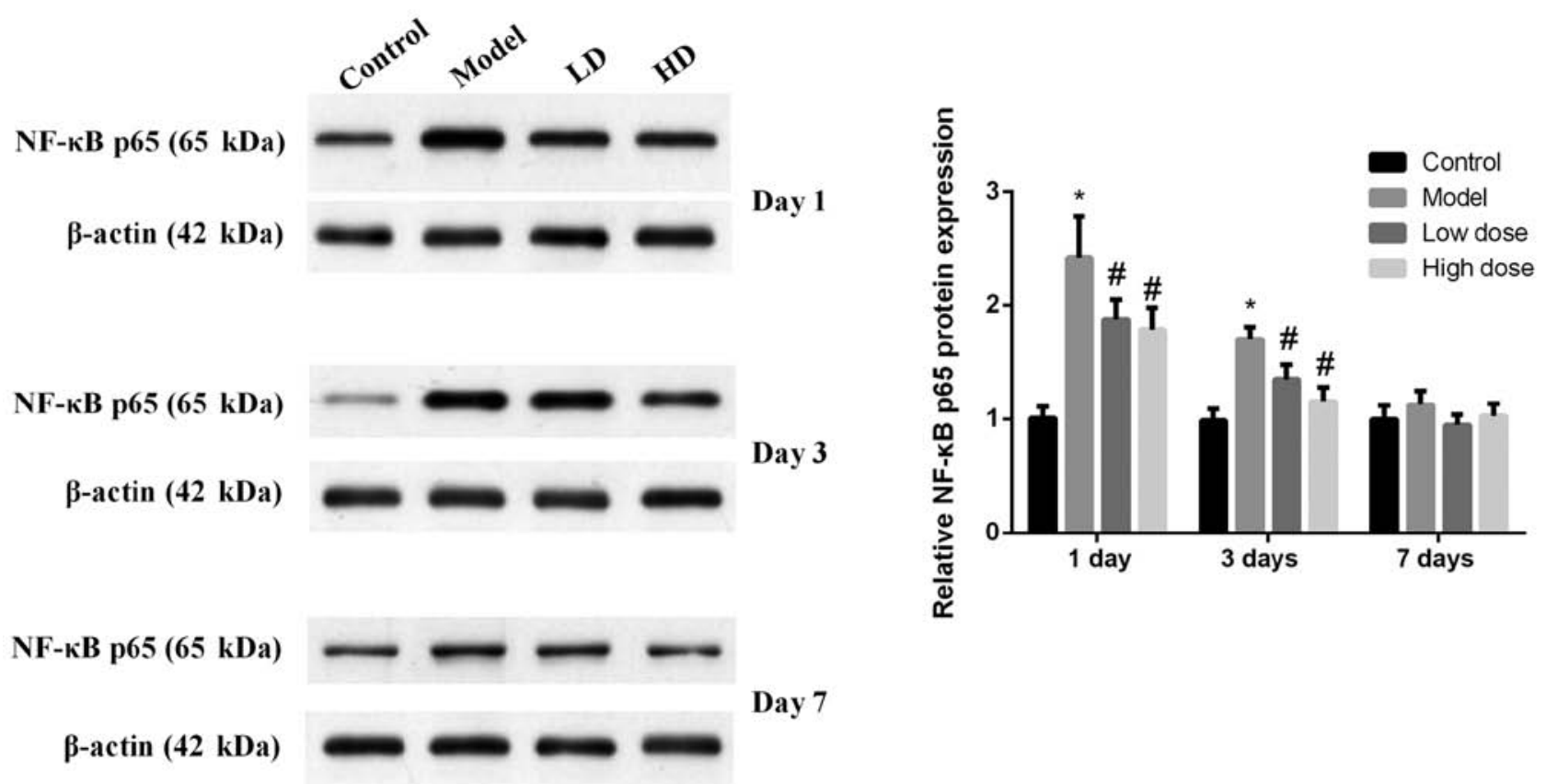

Figure 4. Expression levels of NF- $\mathrm{NB}$ p65 in the hippocampus of rats following surgery. NF- $\mathrm{B}$ p65 expression in the model group was significantly increased compared with the control group at 1 and 3 days after surgery $(\mathrm{P}<0.05)$. The expression levels of $\mathrm{NF}-\kappa \mathrm{B}$ p65 in the high-dose and low-dose groups were significantly decreased compared with those in the model group, and the decrease was more obvious in the high-dose group. At 7 days after surgery, the expression levels of $\mathrm{NF}-\kappa \mathrm{B}$ p 65 remained low and there was no statistically significant difference identified among the groups. ${ }^{\mathrm{P}}<0.05$ vs. control group; ${ }^{\#} \mathrm{P}<0.05$ vs. model group. HD, high-dose; LD, low-dose.

can block the thermal response of the soft palate induced by injection of LPS (24). Additionally, the fever caused by intraperitoneal injection of LPS may be decreased after the vagus nerve is cut (25). These findings suggest that primary sensory nerves may mediate inflammatory factors and indirectly affect the brain tissue. Peripheral inflammatory mediators act inside the brain tissue, causing microglial cells to be rapidly activated, and to release a large number of inflammatory factors (26). In addition, neurons and peripheral lymphocytes entering the brain can release a variety of inflammatory factors, including IL- $1 \beta$, TNF- $\alpha$ and interferon $\gamma$, which, in turn, activate microglia cells and further stimulate signaling transduction pathways, resulting in a cascade of amplified inflammatory responses (27). The inflammatory response in the brain is involved in numerous processes of degenerative alterations in the nervous system. On one hand, inflammation can resist the damage to the body caused by external stimuli; on the other hand, inflammation can damage body tissues through various mechanisms, including degeneration, necrosis, metabolic dysfunction of the parenchymal cells in the inflammatory foci, and mechanical obstruction and compression caused by inflammatory exudates may cause dysfunction of the inflammatory organs. Pain can also affect the active function of the brain (28). Accumulation of a large number of inflammatory mediators and other toxicants in the brain can cause reversible or irreversible damage to the brain tissue, and impaired cognitive function. The hippocampus exhibits widely-expressed receptors for inflammatory factors and is more sensitive to 
A
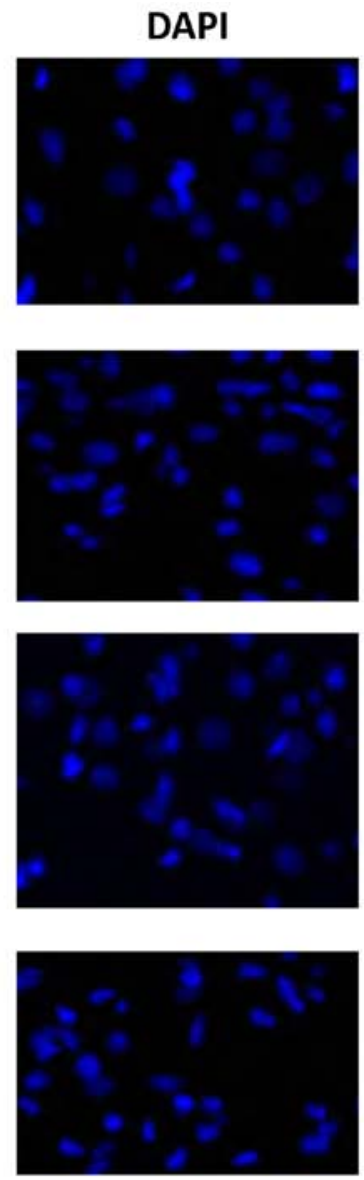

Iba-1
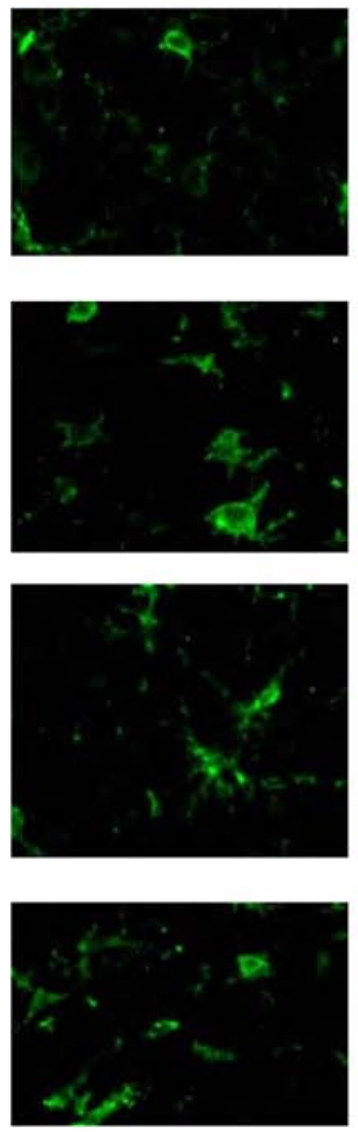

Mixed
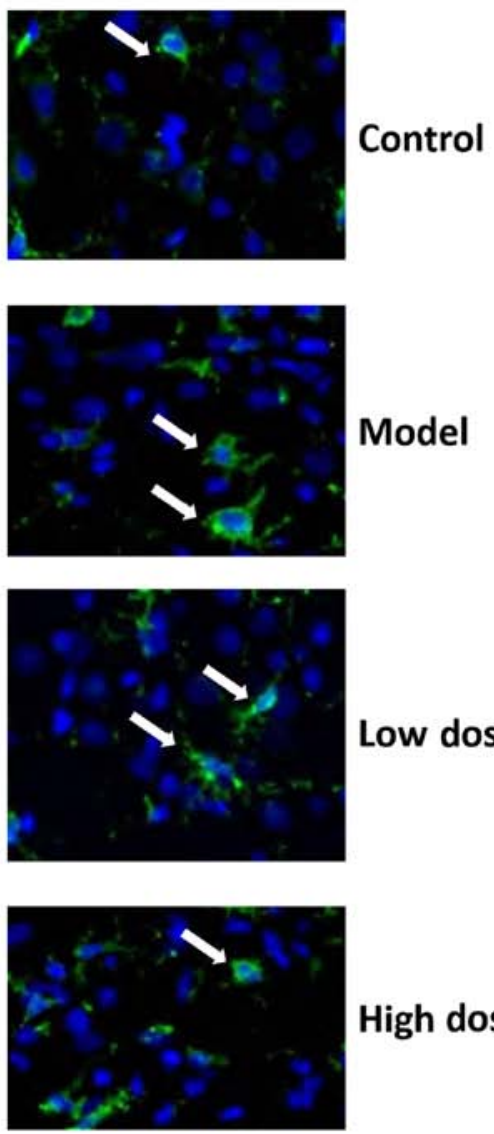

Low dose
High dose

Model

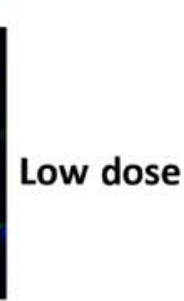

B

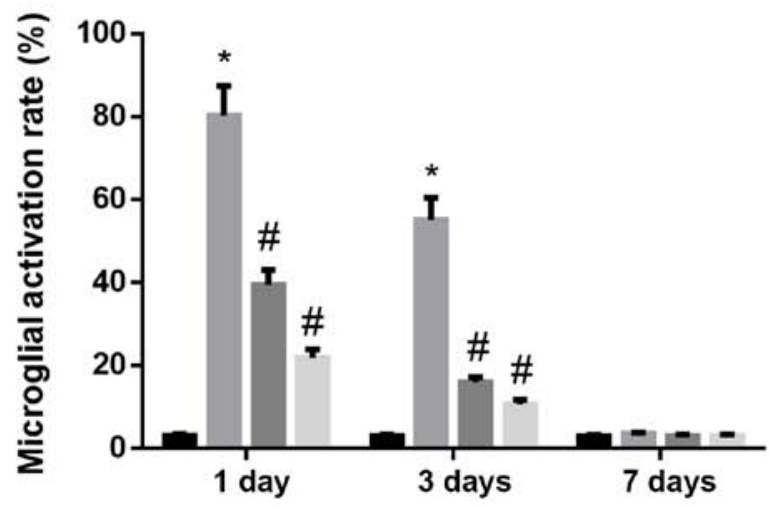

Control

Model

Low dose

High dose

Figure 5. Activation of microglia in the brains of rats following surgery. (A) Immunofluorescence staining of microglia in the hippocampus at 1 day after surgery. Magnification, x400. Arrows indicate activated microglia. (B) Microglial activation rates at 1, 3 and 7 days after surgery. At 1 day after surgery, the microglial cells in the brain tissues of rats in the control group were in an inactive state, cell bodies were small with irregular protrusions. By contrast, the microglial cells in the brain tissues of rats in the model group were large, cell bodies were round and they exhibited an activation state of deformation and phagocytosis. At day 1, the quantification of immunofluorescence staining revealed that the proportion of activated microglial cells was $79.54 \pm 5.52 \%$ in the model group, which was significantly higher than that of the control group $(\mathrm{P}<0.05)$. The activation of microglia in the brain tissue from the high-dose and low-dose anesthesia groups was inhibited, the activation rates were $21.84 \pm 2.03$ and $39.45 \pm 3.55 \%$, respectively, which were significantly lower than that of the model group $(\mathrm{P}<0.05)$. At 3 days after surgery, the activation of microglia in brain tissue was decreased to $55.16 \pm 5.23 \%$ in the model group, to $15.97 \pm 1.26 \%$ in the low-dose and to $10.65 \pm 1.12 \%$ high-dose groups. At 7 days after surgery, there was no statistically significant difference observed in the activation rates of microglial cells in the brain tissues of each group $(\mathrm{P}>0.05)$. These results indicated that microglia were activated by dexmedetomidine. $\mathrm{P}<0.05$ vs. control group; ${ }^{\text {}} \mathrm{P}<0.05$ vs. model group. Iba-1, ionized calcium-binding adapter molecule 1 .

abnormal increases in inflammatory cytokines (29). IL-1 $\beta$ and TNF- $\alpha$ can directly or indirectly penetrate the blood-brain barrier, and they are a class of inflammatory factors with a wide range of biological activities (30). Subsequent to entering the brain, these inflammatory factors cause cell edema, affecting the function of synaptic connections, causing an inflammatory response in the CNS and leading to impairments of cognitive function. If the concentration of IL-1 $\beta$ in the hippocampus is high, it may affect the plasticity of the synapse, and thus affect long-term potentiation, resulting in impaired learning and memory (31). IL-1 $\beta$ and TNF- $\alpha$ can stimulate actin in cells other than intracranial neurons, leading to the regeneration of actin, which serves an important role in the process of neurodegenerative diseases $(32,33)$. In the present study, IL-1 $\beta$ 
and TNF- $\alpha$ expression in rat hippocampi were measured by an ELISA, and NF- $\kappa \mathrm{B}$ p65 expression was measured by western blotting. The results revealed that their expression levels were increased following hepatectomy (for 1-3 days), which was consistent with previous studies $(4,8,34)$. Additionally, the present study demonstrated that dexmedetomidine could significantly reduce their expression levels (between 1 and 3 days), and this reduction was concentration-dependent.

Microglia are macrophage-like cells in the CNS which perform immune surveillance and defense functions in the brain (35). Under normal conditions, the microglia are in a resting state, their cell bodies are small and there are long protrusions surrounding the cells. In this state, microglia lack phagocytic function (36). Microglia can respond to subtle pathological alterations in the nervous system. Microglia can be activated by infection, trauma and ischemia, and perform innate immune functions, including the induction of inflammation, cytotoxic effects and modulation of $\mathrm{T}$ cell responses via antigen presentation (37). Activated microglia can release inflammatory factors, including TNF- $\alpha$, IL-1 $\beta$, IL-6, nitric oxide and prostaglandin E2 (38). These inflammatory factors exhibit a strong toxic effect on neurons and reactivate microglia, aggravating or expanding the inflammatory response of the CNS (39). Therefore, inhibiting the expression of central inflammatory factors may serve a role in improving postoperative cognitive function, and the results of the present study supported this hypothesis. The present study revealed that microglia were activated after hepatectomy and returned to a resting state at 7 days. Dexmedetomidine could significantly reduce the activation of microglia in POCD rats in a concentration-dependent manner. These results indicated that microglia responded to inflammatory alterations and were involved in POCD development, which was consistent with previous studies $(13,35,39)$.

Dexmedetomidine is commonly used as an $\alpha 2$-agonist in clinical practice (8). A previous study reported that the anti-inflammatory effects of dexmedetomidine may be associated with its central sympathomimetic effects and the activation of cholinergic anti-inflammatory pathways (40). However, its mechanism of action has not yet been elucidated. Hoffman et al (41) applied dexmedetomidine to a rat model of cerebral ischemia and found that there is a dose-associated decrease in blood catecholamine content and histopathological improvement in rats, and this improvement is associated with decreased sympathetic nerve activity. Ma et al (34) reported that dexmedetomidine inhibits systemic inflammatory responses and improves survival in septic shock rats. Additionally, Fang et al (42) demonstrated the anti-inflammatory effects of dexmedetomidine in rat spinal cord injury models. In clinical applications, Yang and Hong (43) reported that dexmedetomidine sedation can significantly reduce the expression levels of pro-inflammatory cytokines IL-1 $\beta$, TNF- $\alpha$ and IL- 6 in the plasma in critically ill patients. The present study demonstrated that dexmedetomidine served an anti-inflammatory role, inhibited the expression of IL-1 $\beta$, TNF- $\alpha$ and NF- $\kappa$ B p65, which are crucial for microglial activation and cognitive dysfunction, and that its effect was concentration-dependent. However, during the present study, it was noted that the NF- $\mathrm{B}$ p 65 expression should be assessed by separating the nucleus and cytoplasm; in order to be more exact, a nuclear/cytoplasmic fractionation is required prior to protein quantification. The present study was also limited to observation and simple detection methods. Further studies are required to investigate how dexmedetomidine suppresses inflammation and improves postoperative cognitive function.

In conclusion, the present study found that the intraperitoneal administration of dexmedetomidine may effectively inhibit the release of inflammatory mediators in the CNS and improve the cognitive function of aged rats. Therefore, the present study may provide a potential method for the treatment of POCD in older patients.

\section{Acknowledgements}

Not applicable.

\section{Funding}

No funding was received.

\section{Availability of data and materials}

The datasets used and/or analyzed during the current study are available from the corresponding author on reasonable request.

\section{Authors' contributions}

JQ designed the study. NC and XC analyzed the data and wrote the manuscript. JX performed experiments, including western blotting, ELISA and immunofluorescent staining. CW established the animal model and carried out the behavioral tests. All authors read and approved the final manuscript.

\section{Ethics approval and consent to participate}

All procedures were approved by the Ethics Committee of Zhejiang Hospital.

\section{Patient consent for publication}

Not applicable.

\section{Competing interests}

The authors declare that they have no competing interests.

\section{References}

1. Xu J, Dong H, Qian Q, Zhang X, Wang Y, Jin W and Qian Y: Astrocyte-derived CCL2 participates in surgery-induced cognitive dysfunction and neuroinflammation via evoking microglia activation. Behav Brain Res 332: 145-153, 2017.

2. Berger M, Nadler JW, Browndyke J, Terrando N, Ponnusamy V, Cohen HJ, Whitson HE and Mathew JP: Postoperative cognitive dysfunction: Minding the gaps in our knowledge of a common postoperative complication in the elderly. Anesthesiol Clin 33: 517-550, 2015.

3. Ballard C, Jones E, Gauge N, Aarsland D, Nilsen OB, Saxby BK, Lowery D, Corbett A, Wesnes K, Katsaiti E, et al: Optimised anaesthesia to reduce post operative cognitive decline (POCD) in older patients undergoing elective surgery, a randomised controlled trial. PLoS One 7: e37410, 2012. 
4. Chen J, Buchanan JB, Sparkman NL, Godbout JP, Freund GG and Johnson RW: Neuroinflammation and disruption in working memory in aged mice after acute stimulation of the peripheral innate immune system. Brain Behav Immun 22: 301-311, 2008.

5. Cao XZ, Ma H, Wang JK, Liu F, Wu BY, Tian AY, Wang LL and Tan WF: Postoperative cognitive deficits and neuroinflammation in the hippocampus triggered by surgical trauma are exacerbated in aged rats. Prog Neuropsychopharmacol Biol Psychiatry 34 $1426-1432,2010$

6. Wuri G, Wang DX, Zhou Y and Zhu SN: Effects of surgical stress on long-term memory function in mice of different ages. Acta Anaesthesiol Scand 55: 474-485, 2011.

7. Devasya A and Sarpangala M: Dexmedetomidine: A review of a newer sedative in dentistry. J Clin Pediatr Dent 39: 401-409, 2015.

8. Keating GM: Dexmedetomidine: A review of its use for sedation in the intensive care setting. Drugs 75: 1119-1130, 2015.

9. Celik F, Göcmez C, Kamașak K, Tufek A, Guzel A, Tokgoz O, Firat $\mathrm{U}$ and Evliyaoğlu $\mathrm{O}$ : The comparison of neuroprotective effects of intrathecal dexmedetomidine and metilprednisolone in spinal cord injury. Int J Surg 11: 414-418, 2013.

10. Koca U, Olguner ÇG, Ergür BU, Altekin E, Taşdöğen A, Duru S, Girgin P, Gündüz K, Cilaker Micılı S, Güzeldağ S and Akkuş M: The effects of dexmedetomidine on secondary acute lung and kidney injuries in the rat model of intra-abdominal sepsis. ScientificWorldJournal 2013: 292687, 2013.

11. Yeh CH, Hsieh LP, Lin MC, Wei TS, Lin HC, Chang CC and Hsing $\mathrm{CH}$ : Dexmedetomidine reduces lipopolysaccharide induced neuroinflammation, sickness behavior, and anhedonia. PLoS One 13: e0191070, 2018.

12. Han C, Fu R and Lei W: Beneficial effects of dexmedetomidine on early postoperative cognitive dysfunction in pediatric patients with tonsillectomy. Exp Ther Med 16: 420-426, 2018.

13. Yang Z, Liu Y, Yuan F, Li Z, Huang S, Shen H and Yuan B: Sinomenine inhibits microglia activation and attenuates brain injury in intracerebral hemorrhage. Mol Immunol 60: 109-114, 2014.

14. Frakes AE, Ferraiuolo L, Haidet-Phillips AM, Schmelzer L, Braun L, Miranda CJ, Ladner KJ, Bevan AK, Foust KD, Godbout JP, et al: Microglia induce motor neuron death via the classical NF- $\kappa \mathrm{B}$ pathway in amyotrophic lateral sclerosis. Neuron 81: 1009-1023, 2014.

15. Park J, Min JS, Kim B, Chae UB, Yun JW, Choi MS, Kong IK, Chang KT and Lee DS: Mitochondrial ROS govern the LPS-induced pro-inflammatory response in microglia cells by regulating MAPK and NF- $\kappa$ B pathways. Neurosci Lett 584: 191-196, 2015.

16. Chen SQ, Wang PJ, Ten GJ, Zhan W, Li MH and Zang FC: Role of myo-inositol by magnetic resonance spectroscopy in early diagnosis of Alzheimer's disease in APP/PS1 transgenic mice. Dement Geriatr Cogn Disord 28: 558-566, 2009.

17. Zhu YS, Xiong YF, Luo FQ and Min J: Dexmedetomidine protects rats from postoperative cognitive dysfunction via regulating the $\mathrm{GABA}_{\mathrm{B}}$ R-mediated cAMP-PKA-CREB signaling pathway. Neuropathology 39: 30-38, 2019.

18. Xu Z, Wang D, Zhou Z, Chen Q, Zhang D, Chen S, Jiang H, Jia C and Liu X: Dexmedetomidine attenuates renal and myocardial Ischemia/Reperfusion injury in a dose-dependent manner by inhibiting inflammatory response. Ann Clin Lab Sci 49: 31-35, 2019.

19. Tucker LB, Fu AH and McCabe JT: Performance of male and female $\mathrm{C} 57 \mathrm{BL} / 6 \mathrm{~J}$ mice on motor and cognitive tasks commonly used in pre-clinical traumatic brain injury research. J Neurotrauma 33: 880-894, 2016.

20. Shoham S, Linial M and Weinstock M: Age-induced spatial memory deficits in rats are correlated with specific brain region alterations in microglial morphology and gene expression. J Neuroimmune Pharmacol 14: 251-262, 2019.

21. Hsu WC, Yu CH, Kung WM and Huang KF: Enhancement of matrix metalloproteinases 2 and 9 accompanied with neurogenesis following collagen glycosaminoglycan matrix implantation after surgical brain injury. Neural Regen Res 13: 1007-1012, 2018.

22. Lord JM, Midwinter MJ, Chen YF, Belli A, Brohi K, Kovacs EJ, Koenderman L, Kubes P and Lilford RJ: The systemic immune response to trauma: An overview of pathophysiology and treatment. Lancet 384: 1455-1465, 2014.

23. Podjaski C, Alvarez JI, Bourbonniere L, Larouche S, Terouz S, Bin JM, Lécuyer MA, Saint-Laurent O, Larochelle C, Darlington PJ, et al: Netrin 1 regulates blood-brain barrier function and neuroinflammation. Brain 138: 1598-1612, 2015.
24. Romeo HE, Tio DL and Taylor AN: Effects of glossopharyngeal nerve transection on central and peripheral cytokines and serum corticosterone induced by localized inflammation. J Neuroimmunol 136: 104-111, 2003.

25. Le Maitre E, Revathikumar P, Estelius J and Lampa J: Increased recovery time and decreased LPS administration to study the vagus nerve stimulation mechanisms in limited inflammatory responses. J Vis Exp: Mar 29, 2017. doi: 10.3791/54890.

26. Hoogland IC, Houbolt C, van Westerloo DJ, van Gool WA and van de Beek D: Systemic inflammation and microglial activation: Systematic review of animal experiments. J Neuroinflammation 12: $114,2015$.

27. Gyoneva S, Davalos D, Biswas D, Swanger SA, Garnier-Amblard E, Loth F, Akassoglou K and Traynelis SF: Systemic inflammation regulates microglial responses to tissue damage in vivo. Glia 62: $1345-1360,2014$.

28. Kang YC, Zhang L, Su Y, Li Y, Ren WL and Wei WS: MicroRNA-26b regulates the microglial inflammatory response in Hypoxia/Ischemia and affects the development of vascular cognitive impairment. Front Cell Neurosci 12: 154, 2018.

29. Fakhoury M: Role of immunity and inflammation in the pathophysiology of neurodegenerative diseases. Neurodegener Dis 15: 63-69, 2015.

30. Kim DW, Lee JC, Cho JH, Park JH, Ahn JH, Chen BH, Shin BN, Tae HJ, Seo JY, Cho JH, et al: Neuroprotection of ischemic preconditioning is mediated by Anti-inflammatory, Not Pro-inflammatory, Cytokines in the Gerbil hippocampus induced by a subsequent lethal transient cerebral ischemia. Neurochem Res 40: 1984-1995, 2015.

31. Loftis JM, Valerio J, Taylor J, Huang E, Hudson R, Taylor-Young P, Chang M, Ho SB, Dieperink E, Miranda JL and Hauser P: S100B and inflammatory cytokine levels in blood as potential markers of blood-brain barrier damage and psychiatric impairment in comorbid hepatitis $\mathrm{C}$ viral infection and alcohol use disorder. Alcohol Clin Exp Res: Jun 28, 2018. doi: 10.1111/acer.13796 (Epub ahead of print).

32. Farbood Y, Sarkaki A, Dianat M, Khodadadi A, Haddad MK and Mashhadizadeh S: Ellagic acid prevents cognitive and hippocampal long-term potentiation deficits and brain inflammation in rat with traumatic brain injury. Life Sci 124: 120-127, 2015.

33. Amor S, Peferoen LA, Vogel DY, Breur M, van der Valk P, Baker D and van Noort JM: Inflammation in neurodegenerative diseases-an update. Immunology 142: 151-166, 2014

34. Ma Y, Yu XY and Wang Y: Dose-related effects of dexmedetomidine on immunomodulation and mortality to septic shock in rats. World J Emerg Med 9: 56-63, 2018.

35. Suzumura A: Neuron-microglia interaction in neuroinflammation. Curr Protein Pept Sci 14: 16-20, 2013.

36. Bogie JF, Stinissen P and Hendriks JJ: Macrophage subsets and microglia in multiple sclerosis. Acta Neuropathol 128: 191-213, 2014.

37. Li C, Chen T, Zhou H, Zhang C, Feng Y, Tang F, Hoi MP, He C, Zheng Y and Lee SM: Schisantherin A attenuates neuroinflammation in activated microglia: Role of Nrf2 activation through ERK phosphorylation. Cell Physiol Biochem 47: 1769-1784, 2018.

38. Peng J, Zhang P, Zheng H, Ren YQ and Yan H: Dexmedetomidine reduces hippocampal microglia inflammatory response induced by surgical injury through inhibiting NLRP3. Chin J Traumatol 22: 161-165, 2019.

39. Li JW, Zong Y, Cao XP, Tan L and Tan L: Microglial priming in Alzheimer's disease. Ann Transl Med 6: 176, 2018.

40. Daskalopoulos EP, Malliou F, Rentesi G, Marselos M, Lang MA and Konstandi M: Stress is a critical player in CYP3A, CYP2C, and CYP2D regulation: Role of adrenergic receptor signaling pathways. Am J Physiol Endocrinol Metab 303: E40-E54, 2012.

41. Hoffman WE, Kochs E, Werner C, Thomas C and Albrecht RF Dexmedetomidine improves neurologic outcome from incomplete ischemia in the rat. Reversal by the alpha 2 -adrenergic antagonist atipamezole. Anesthesiology 75: 328-332, 1991.

42. Fang B, Li XQ, Bi B, Tan WF, Liu G, Zhang Y and Ma H: Dexmedetomidine attenuates blood-spinal cord barrier disruption induced by spinal cord ischemia reperfusion injury in rats. Cell Physiol Biochem 36: 373-383, 2015.

43. Yang D and Hong JH: Dexmedetomidine modulates Histamine-induced $\mathrm{Ca}(2+)$ Signaling and Pro-inflammatory cytokine expression. Korean J Physiol Pharmacol 19: 413-420, 2015.

This work is licensed under a Creative Commons

Attribution-NonCommercial-NoDerivatives 4.0 International (CC BY-NC-ND 4.0) License. 